\title{
Asthma and comorbid medical illness
}

\author{
M. Cazzola*\#, L. Calzetta*,\#, G. Bettoncelli", L. Novelli*, C. Cricelli` and P. Rogliani*
}

ABSTRACT: Asthma is associated with several comorbidities but the magnitude of the association has not been clearly defined. We aimed to examine the relationship between asthma and comorbidities using information obtained from the Health Search Database (HSD) owned by the Italian College of General Practitioners (Società Italiana Medici Generici, Florence, Italy).

We conducted a population-based retrospective study using information obtained from the HSD. The software system used codes of all the diagnostic records using the 9th revision of the International Classification of Diseases.

Asthma appeared to be weakly associated with cardiovascular and hypertensive diseases. Intriguingly, the odds ratio of acute or old myocardial infarction was 0.84 (95\% $\mathrm{Cl} 0.77-0.91)$. Asthma was also weakly associated with depression, diabetes mellitus, dyslipidaemia, osteoporosis and rhinosinusitis. In contrast, it was strongly associated with gastro-oesophageal reflux disease (GORD) and, particularly, allergic rhinitis. Age did not influence the association of asthma with comorbidities whereas sex had a different impact according to the specific comorbidity.

Our results indicate that asthma is weakly associated with several comorbidities, whereas its association with allergic rhinitis or GORD is stronger.

KEYWORDS: Age, asthma, comorbidities, sex

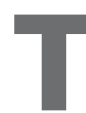
raditionally, comorbidity has been defined as a disease co-existing with the primary disease of interest [1]. Comorbidities, such as ischaemic heart disease, heart failure, osteoporosis, normocytic anaemia, lung cancer, depression and diabetes, may frequently occur in patients with chronic obstructive pulmonary disease (COPD) [2-4]. It has been suggested that comorbidities in COPD should be defined as the presence of one or more distinct disorders (or diseases) in addition to COPD, regardless of whether the comorbid conditions are directly or indirectly related to COPD, and a distinct disorder or disease is not part of the spectrum of the natural history of COPD [1].

In addition, asthma has been proposed to be associated with several comorbidities [5]. In fact, some reports suggest that various comorbid conditions occur more frequently than expected with asthma, with some being associated with poor control and a differential response to therapy options, although their role in the clinical expression of asthma has not been fully elucidated [6]. Unfortunately, the prevalence of comorbidities in asthmatic patients varies tremendously between studies [6], probably because we still do not know whether the comorbid conditions are directly or indirectly related to asthma since they are generally not considered part of the spectrum of the natural history of asthma.
In the present study, we aimed to examine the relationship between asthma and its comorbidities, and determine the relative magnitude of association of asthma with each of the most common comorbidities, using information obtained from the Health Search Database (HSD) owned by the Società Italiana Medici Generici (the Italian College of General Practitioners, Florence, Italy). The HSD stores information on $\sim 1.5 \%$ of the total Italian population served by general practitioners (GPs).

\section{PATIENTS AND METHODS}

\section{Collection of information}

Primary-care data were obtained from the HSD of the Società Italiana Medici Generici. Established in 1998 with the primary aim of carrying out observational studies on the incidence and prevalence of chronic diseases, with the input of GPs who agreed to participate on a voluntary basis, the HSD contains information provided by $>650$ Italian GPs and includes a total of $>900,000$ individuals aged $>14$ yrs (since 1978 the Italian National Health Service has provided paediatric primary care to children aged 0-14 yrs through the use of community-based paediatricians).

After intensive training, all participating GPs used specially designed software to record data during their normal daily clinical practice (Millewin S.r.L, Florence, Italy). The software system codes all the diagnostic records by using
AFFILIATIONS

*Dept of Internal Medicine, University of Rome Tor Vergata, \#Dept of Pulmonary Rehabilitation, IRCCS, San Raffaele Pisana Hospital, Rome, and

'Health Search Institute, Italian College of General Practitioners, Florence, Italy.

\section{CORRESPONDENCE}

M. Cazzola

Cattedra di Malattie Respiratorie, Dipartimento di Medicina Interna Università di Roma Tor Vergata Via Montpellier 1 IT-00133 Rome Italy E-mail: mario.cazzola@uniroma2.it

Received:

Sept 012010

Accepted after revision: Nov 302010

First published online: Dec 222010 
the International Classification of Diseases 9th revision clinical modification (ICD-9-CM) [7]. Prescription records were also coded according to the anatomical therapeutic chemical classification system. Data were recorded in the HSD with the consent of the patient, lawfully stored, managed according to privacy rules and can be used for scientific studies without any further authorisation from an ethics committee.

It is well known that before using any data resource, particularly one that is based on computer records, it is necessary to determine the quality and completeness of the available information. Therefore, data were subjected to a range of quality checks. Any variations within agreed ranges were investigated and submitted to each participating GP. Physicians who failed to meet standard quality criteria were not considered for epidemiological studies [8].

We must highlight that previous comparative analyses have demonstrated the validity of the information gathered in the HSD [9].

\section{Ascertainment of asthma and comorbidities}

Cases of asthma were identified on the basis of the ICD-9 code 493. All cases identified on the basis of the ICD-9 codes 491, 492 and 496 were not considered in this study. The number of patients classified on the basis of the ICD-9 code 493.2 was very small and for this reason, as well as to avoid a statistical bias, we have not considered it separately. Unfortunately, we do not have information in our database related to temporality.

Using the ICD-9 codes, we regarded the following as cases of chronic specific comorbidities: ischaemic heart disease (410$414)$; diseases of the pulmonary circulation (415-417); other forms of heart disease (420-429); cardiac arrhythmia (427); heart failure (428); hypertensive disease (401-405); diabetes mellitus (250); dyslipidaemia (272.4); osteoporosis (733.0); neurotic disorders, personality disorders and other non-psychotic mental disorders (300-316); depressive disorders not classified (311); gastro-oesophageal reflux disease (GORD; 530.81); allergic rhinitis (477); and sinusitis (473.9).

\section{Analysis}

The study was carried out using a cross-sectional and observational field method. It allowed for description of the situation at the time of data collection (the end of December 2009) and allowed for determination of the prevalence of investigated outcomes. The studied population was divided into several clusters: age (15-34, 35-44, 45-54, 55-64, 65-74, 7584 and $\geqslant 85 \mathrm{yrs}$ ), sex (male and female) and comorbidities.

We evaluated the odds ratio (OR), which was chosen instead of relative risk because asthma has a prevalence that is $<10 \%$ $[10,11]$. Positive and negative OR values represent a co-relative positive and negative association between evaluated variables. Data were adjusted for sex and age using the Mantel-Haenszel method, which permits a precision-based estimate of the OR. Furthermore, if the prevalence of comorbidities was $>10 \%$ and the OR was $>2.5$ or $<0.5$, correction of the OR was performed $[10,11]$. In order to calculate statistical and epidemiological values, computer software programs GraphPad Prism (GraphPad Software, La Jolla, CA, USA), SPSS (SPSS Inc., Chicago, IL, USA) and OpenEpi version 2.3.1 (Open Source
Epidemiologic Statistics for Public Health, www.openepi.com/ OE2.3/Menu/OpenEpiMenu.htm) were used.

\section{RESULTS}

We identified a population of 909,638 individuals $(429,962$ males and 479,676 females) aged $>14$ yrs and registered in the HSD at the end of December 2009. There were 55,500 (6.1\%) individuals suffering from asthma. The prevalence of asthma was greater in females than in males with a male to female ratio of 0.83 , and there was a slightly decreased prevalence with age in both males and females.

\section{Prevalence of comorbidities}

Table 1 shows the demographic characteristics of the study population by age, sex, geographic area distribution and asthma prevalence. The individuals who were not identified on the basis of ICD-9 code 493 were considered "nonasthmatics".

Table 2 describes the observed prevalence of comorbidities in the study population. The pooled prevalence of cardiovascular morbidity (acute or old myocardial infarction, angina and coronary disease, cardiac arrhythmia, heart failure, cerebrovascular disease, other heart disease or pulmonary embolism) was $11.6 \%$ in the general population and $11.1 \%$ in asthmatics whereas that of hypertensive disease was $25.7 \%$ in the general population and $28.3 \%$ in asthmatics. Overall, $28.7 \%$ of the general population and $37.8 \%$ of the asthmatic population were identified with a recorded code for non-psychotic mental disorders, including depressive disorders not classified elsewhere. The prevalence of diabetes mellitus was $7.8 \%$ in the general population and $8.4 \%$ in asthma, whereas that of dyslipidaemia was $3.2 \%$ in the general population and $4.2 \%$ in asthma. $9.8 \%$ of the general population and $16.6 \%$ of the asthmatics suffered from GORD. In the general population, the prevalence of allergic rhinitis was $6.2 \%$ and that of rhinosinusitis $3.6 \%$, whereas between asthmatic subjects $18.5 \%$ were also identified with a code for allergic rhinitis and $5.7 \%$ with a code for rhinosinusitis. The prevalence of osteoporosis was $7.6 \%$ in the general population and $10.3 \%$ in asthma.

\section{Association between asthma and comorbidities}

Figure 1 shows ORs for the association between asthma and individual chronic conditions. Asthma appeared to be weakly associated with cardiovascular and hypertensive diseases. Intriguingly, the OR of acute or old myocardial infarction was 0.84 (95\% CI 0.77-0.91). Asthma was also weakly associated with depression, diabetes mellitus, dyslipidaemia, osteoporosis and rhinosinusitis. In contrast, it was strongly associated with GORD and, particularly, allergic rhinitis.

\section{Impact of sex on comorbidities in asthma}

Being female slightly increased the association of all cardiovascular diseases, mainly heart failure, but not angina, coronary disease and acute or old myocardial infarction, with asthma (fig. 1). In males, there was no association between asthma and acute or old myocardial infarction; moreover, in males, asthma was not associated with hypertensive disease. However, in contrast to females, males presented with an association between asthma and angina and coronary disease. 
TABLE 1 Demographic characteristics of study population by age, sex, geographic area distribution and asthma prevalence

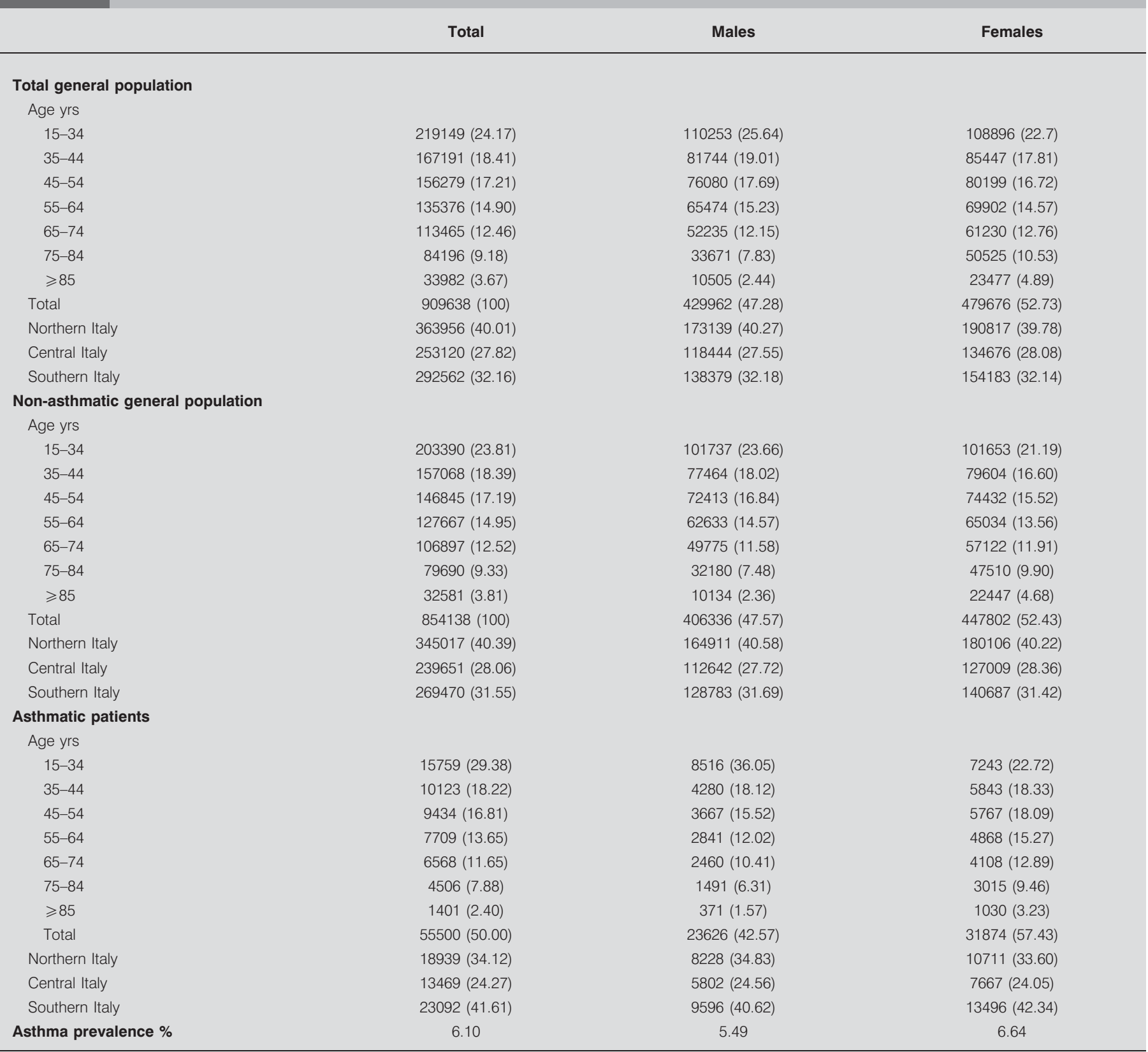

Data are presented as n (\%), unless otherwise stated

In females, the association between diabetes mellitus, dyslipidaemia, osteoporosis, depression, psychiatric disorders and GORD and asthma was stronger than in males. In males there was no association between asthma and diabetes whereas the association between asthma and allergic rhinitis and rhinosinusitis was stronger than in females.

\section{Impact of age on comorbidities in asthma}

Figure 2 shows the ORs of various chronic comorbidities in individuals with asthma across different age groups. The prevalence of cardiovascular diseases and hypertensive disease increased with age, but we did not observe significant OR value increases with age. In addition, for all other comorbidities, increasing age resulted in a progressive rise in prevalence but changes in age did not really increase the association. In contrast, the prevalence of allergic rhinitis decreased with the increase in age but, again, the different age clusters did not affect its association with asthma.

\section{DISCUSSION}

\section{Interpretation of association between asthma and comorbidities}

Analysis of data in the HSD allowed us to confirm that allergic rhinitis and GORD are comorbidities that frequently co-exist 
TABLE 2 Demographic characteristics of study population by prevalence of comorbidities

\begin{tabular}{|c|c|c|c|c|c|c|c|c|c|}
\hline & Total & Males & Females & Total & Males & Females & Total & Males & Females \\
\hline Ischaemic cardiopathy & 2.05 & 2.58 & 1.57 & 2.03 & 2.57 & 1.53 & 2.40 & 2.63 & 2.23 \\
\hline Myocardial infarction & 1.24 & 1.91 & 0.64 & 1.25 & 1.93 & 0.64 & 1.05 & 1.58 & 0.66 \\
\hline Cardiac arrhythmia & 5.30 & 5.02 & 5.55 & 5.21 & 4.96 & 5.43 & 6.70 & 5.90 & 7.29 \\
\hline Cerebrovascular disease & 1.64 & 1.43 & 1.83 & 1.63 & 1.43 & 1.81 & 1.88 & 1.44 & 2.21 \\
\hline Other heart disease & 0.18 & 0.19 & 0.18 & 0.18 & 0.18 & 0.17 & 0.28 & 0.27 & 0.28 \\
\hline Pulmonary embolism & 0.17 & 0.16 & 0.19 & 0.17 & 0.15 & 0.19 & 0.26 & 0.21 & 0.29 \\
\hline Allergic rhinitis & 6.18 & 6.13 & 6.22 & 5.38 & 5.35 & 5.41 & 18.53 & 19.67 & 17.68 \\
\hline Rhinosinusitis & 3.61 & 2.97 & 4.18 & 3.47 & 2.86 & 4.02 & 5.74 & 4.92 & 6.34 \\
\hline Diabetes mellitus & 7.82 & 8.49 & 7.22 & 7.78 & 8.54 & 7.10 & 8.36 & 7.68 & 8.87 \\
\hline Depression & 4.48 & 2.79 & 5.99 & 4.36 & 2.75 & 5.83 & 6.21 & 3.48 & 8.23 \\
\hline Gastro-oesophageal reflux & 9.85 & 8.73 & 10.85 & 9.41 & 8.44 & 10.29 & 16.56 & 18.74 & 13.63 \\
\hline
\end{tabular}

with asthma. We must stress that our database only allowed us to examine the association of comorbidities and asthma in adults. A recent review of the published evidence on comorbidities of asthma during childhood described overweight, allergic rhinitis, dysfunctional breathing, depressive disorders and, perhaps, GORD as the most frequent comorbidities in children [12].

The majority of patients with asthma present with symptoms of seasonal or perennial allergic rhinitis [13]. A cross-sectional, observational study conducted in France by GPs to describe the frequency and severity of allergic rhinitis according to the severity of the asthmatic disease, suggested that allergic rhinitis was associated with more severe asthma [14]. We cannot confirm or deny this finding because the HSD does not offer any real indication on asthma severity. Asthma and allergic rhinitis share similar triggers and pathophysiology, characterised by similar inflammatory cell infiltrates [15]. It has been suggested a central mechanism behind the link with eosinophil precursors emanating from the bone marrow in response to triggers migrating not only to the site of stimulation, such as the nasal mucosa, but also to other sites within the one airway, including the lower respiratory tract [16]. Autonomic imbalance also plays a role in both conditions
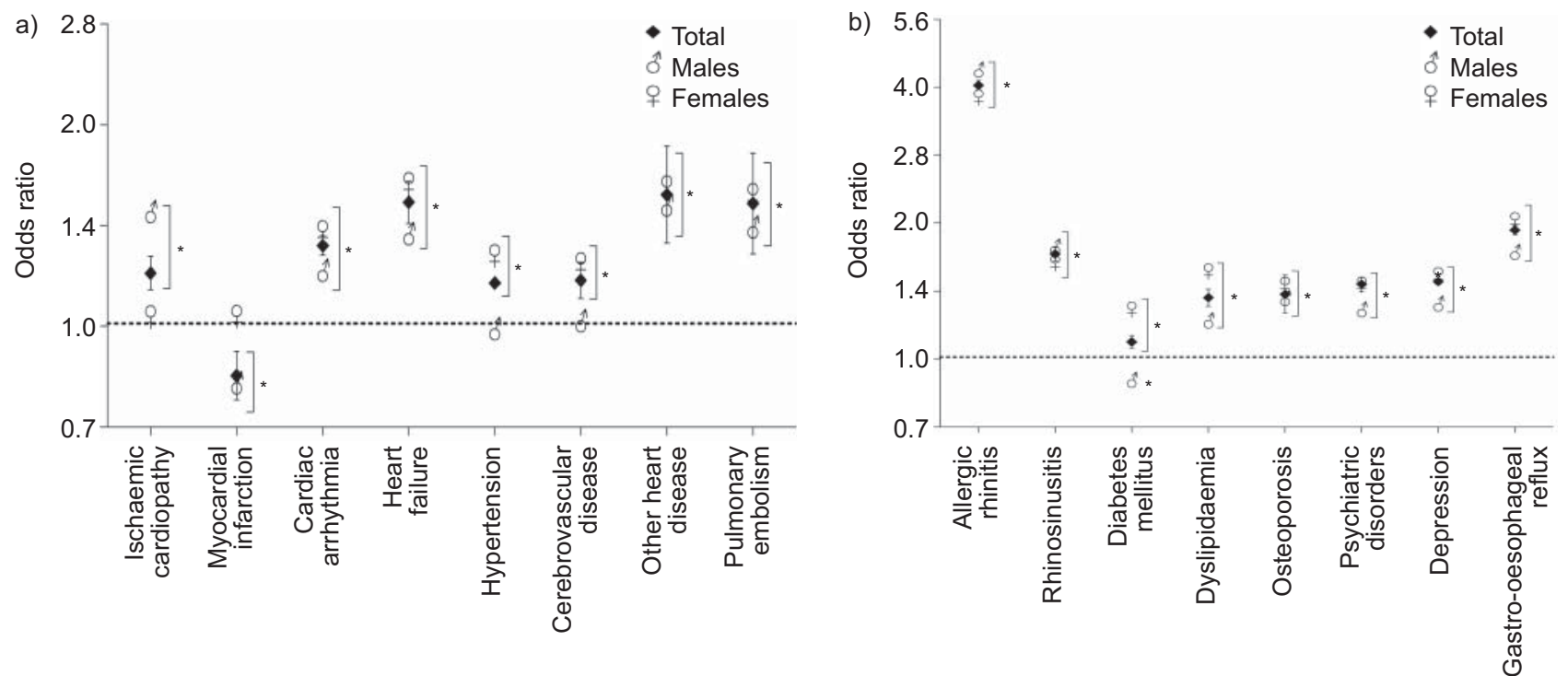

FIGURE 1. Adjusted odds ratios and $95 \%$ confidence intervals of comorbidities in asthma. Total is adjusted for age and sex. Males and females are adjusted for age. a) Cardiovascular comorbidities and b) other comorbidities. *: $p<0.05$ versus non-asthmatic population. 

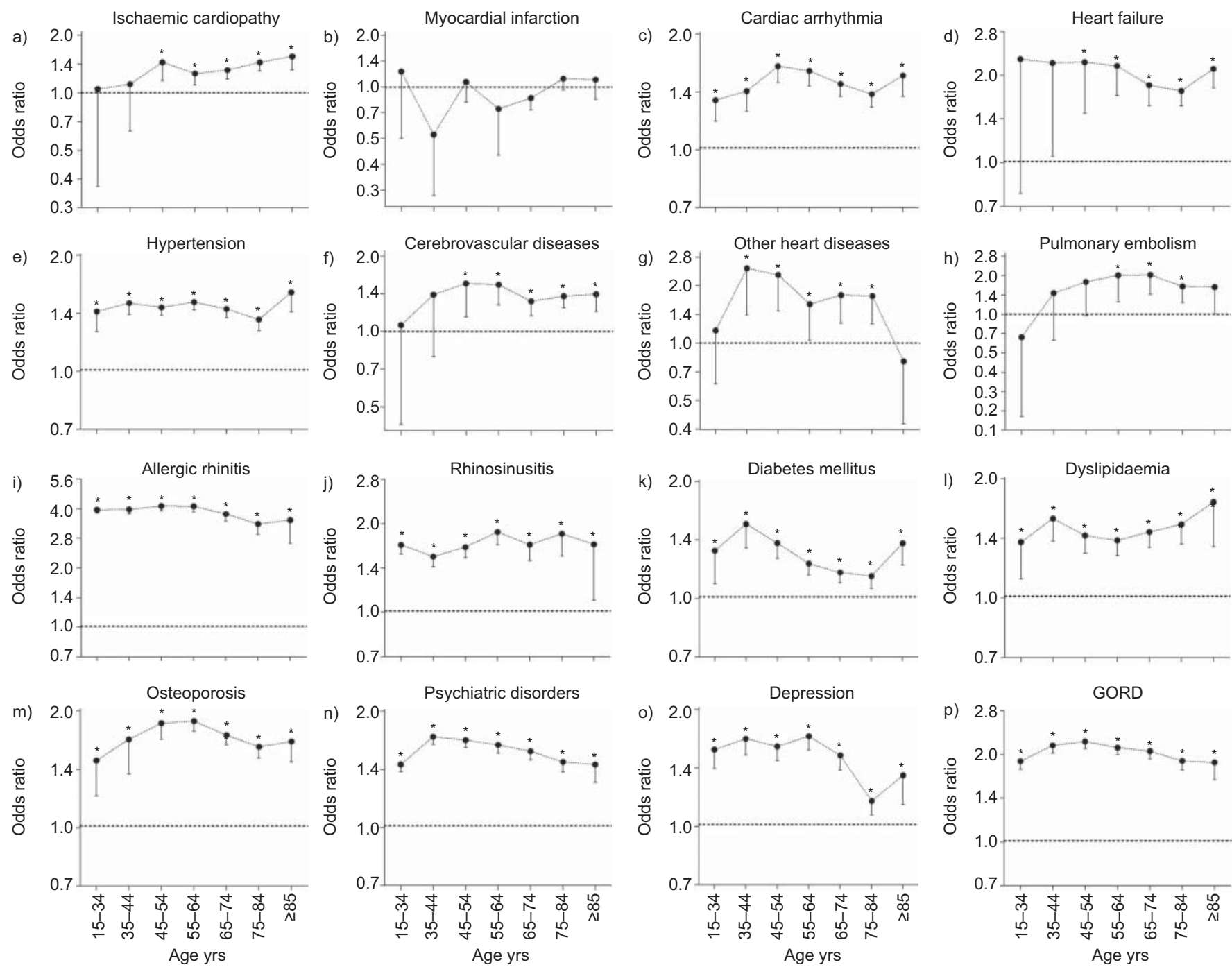

FIGURE 2. Adjusted odds ratios and $95 \%$ confidence interval of comorbidities in asthma in different strata of age. Values were adjusted for sex. GORD: gastrooesophageal reflux. ${ }^{*}: p<0.05$ versus comorbidities in non-asthmatic population.

via changes in neural tone to effector tissues, release of neuropeptides and interplay with cellular recruitment [17]. Increased lower airway exposure to airborne contaminants from mouth breathing and increased need for conditioning the inspired air are two other suggested mechanisms of nose-lung interaction $[15,18]$.

Our results also show a link between asthma and rhinosinusitis. This finding is in agreement with the fact that allergic rhinitis is a risk factor for sinusitis, and asthmatics are known to be at increased risk for sinusitis [6]. However, contrary to the findings in a large cohort of asthmatics, which documented that females were associated with an increased risk of allergic rhinitis and sinusitis [19], in our population both allergic rhinitis and rhinosinusitis presented a stronger association with asthma in males than in females.

Patients with asthma are also at a significantly increased risk of developing GORD [20]. It has been suggested that the altered respiratory physiology in asthma patients may predispose them towards gastro-oesophageal reflux [21]. Respiratory obstruction can result in negative pleural pressures, increasing the pressure gradient between the thorax and abdominal cavity, thus promoting reflux [21]. We cannot confirm this hypothesis because our database does not offer an indication on the temporal sequence relationship between the two diseases. However, whatever the case may be, our data indicate that the different clusters of age are not associated in a different manner with GORD.

We believe that the most important finding of the present study is that asthma is only weakly associated with cardiovascular and hypertensive diseases, contrary to what is observed in patients with COPD [22]. This might question the importance of the systemic inflammation in asthma, as recent studies have suggested a connection between comorbidities and systemic inflammation in COPD [2-4]. In particular, our data indicate that in asthmatic patients there is no real association of their respiratory disease with acute or old myocardial infarction. Moreover, angina and coronary disease are associated with asthma mainly in males, and age does not seem to influence the association between asthma and 
cardiovascular and hypertensive diseases. This conclusion is in agreement with the documentation that asthma is not a risk factor for coronary heart disease in middle-aged adults [23]. Nonetheless, some reports have suggested that subjects with severe asthma, especially females, have a significant, increased risk of ischaemic heart disease [24]. We are unable to explain why there is a significant association between asthma and angina and coronary disease but not myocardial infarction in males, although the prevalence of angina, coronary disease and myocardial infarction in asthmatic males was similar to that in males in the non-asthmatic population. It is likely that the low number of events may have generated information that larger numbers could deny, although again we must emphasise the large size of our sample. In any case, the increased ORs for cardiac arrhythmia and heart failure, as well as other heart diseases, may suggest that the consequences of those diseases on the lung may have been confounded with asthma. However, we must stress that we have only considered comorbidities that were present in the same patient simultaneously with the diagnosis of asthma.

In the present study, we have documented that asthma is only associated with cerebrovascular disease in females and in a weak manner. In an attempt to explain the higher prevalence of cerebrovascular disease in asthmatic females, ONUFRAK et al. [25] provided evidence that adult-onset asthma is associated with carotid atherosclerosis among females but not among males. In contrast, childhood-onset asthma was not significantly associated with atherosclerosis among females or males. Our database does not indicate whether our patients were suffering from childhood- or adult-onset asthma. Consequently, we cannot confirm or deny the different distribution of risk between the sexes related to the different time of onset of asthma.

Our data also showed that non-psychotic mental disorders, including depressive disorders, have a high prevalence in asthmatic patients, indicating that these disorders frequently co-occur. Various studies have investigated whether individuals with asthma are more likely to be depressed than those without asthma, and while a number of studies have supported this hypothesis, other investigators have not found such evidence [26]. In response to the differing findings in regard to this issue, ADAMS et al. [27] theorised that differing sampling techniques may have been related to the discrepancies between findings. Mechanisms of the associations between asthma and mental disorders remain unknown. It has been suggested that there is a causal link between asthma and anxiety disorders; however, increasingly, evidence supports the possibility that one or more outside factors, either environmental or genetic, may influence the risk of both asthma and anxiety disorders [28]. Potential factors that could play a role in causal mechanisms for the relationship between asthma and anxiety disorders include increased levels of anxiety associated with fear of the next asthma attack, the anxiogenic properties of asthma medications, hyperventilation associated with panic attacks, and poor adherence to asthma treatment in patients with psychiatric diagnoses.

The observed association between asthma and diabetes mellitus, at least in females, also deserves attention because it is a finding that contrasts with several reports in the literature.
In an Australian cross-sectional analysis of a representative population of adults, asthma was not a significant risk factor for diabetes [29] and, moreover, no significant association between asthma and risk of type 2 diabetes was found in females [30]. The lack of association between diabetes and asthma, at least in males, is contrary to what is observed in COPD, and may suggest a link to the different pattern of inflammation in asthma compared with COPD [31].

It is intriguing that we have also observed an association between asthma and dyslipidaemia, with a higher OR in females. There are speculative data that suggest that alterations in the type or amount of lipids in the blood could have effects that promote or exacerbate asthma [32]. It has been suggested that leptin might be involved in the pathogenesis of asthma in overweight children and that interferon- $\gamma$ might be a pathway in the process of leptin-induced inflammation [33, 34]. A more recent study indicated a higher prevalence of asthma in children with high serum cholesterol [35]. The study was very small $(n=188)$ and has not yet been reproduced.

Our results confirm that dyslipidaemia, GORD or type 2 diabetes, which are comorbidities of obesity, are associated with asthma. It has been proposed that comorbidities of obesity may provoke or worsen asthma [32]. We must mention that the HSD indicates a clear relationship between body mass index (BMI) and asthma (data not shown), suggesting that the prevalence of asthma is increased in proportion to BMI. We must also stress that obese asthma patients do not demonstrate increased cellular airway inflammation, possibly because of the presence of a non-T-helper 2 cytokine-driven, nonallergic asthma phenotype. Comorbidities, such as GORD, may contribute to the presentation of asthma in the obese and, intriguingly, there is also increasing evidence that cytokines secreted by adipose tissue cause systemic inflammation [36].

Although in our population there was a high prevalence of osteoporosis among females, the evidence that the ORs of the two sexes were similar is worth mentionimg, considering the observed lack of association due to age. To the best of our knowledge, this is the first report that indicates that sex is not an important factor in the link between asthma and osteoporosis. However, we must mention that osteoporosis in males is substantially under-diagnosed, under-treated, underreported and, particularly, inadequately researched [37]. This could justify the reported higher prevalence of osteoporosis among females, and also explain why the majority of studies have looked at the link between asthma and osteoporosis only in females with contrasting results.

\section{Strengths and limitations of the study}

We believe that our results are interesting because they are from a large, validated, population-based database that illustrates what can be observed in real life and what can be considered representative of the general population. This is the major strength of the present study. However, we must admit that the present study has important limitations, many of which are common to retrospective database analyses. These limitations might be mainly associated with a bias towards the selection of more highly motivated or better organised practices. Consequently, our findings may be an overestimate of the general picture. Nonetheless, it is well known that patients vary in their 
use of healthcare and it is accepted that a significant proportion of patients who are ill might not consult their doctor and this can lead to underestimation of the problem. Moreover, we must emphasise that HSD data relate to those diagnoses for which a relevant medication is prescribed. Thus, for example, a diabetic patient who is controlled with diet therapy will not be detected. This limitation may underestimate the true prevalence of the disease, but it ensures that the condition is currently active and the diagnosis is severe enough to require pharmacotherapy.

We must also highlight that there may have been misclassification when our GPs used specific diagnostic categories and this was probably because there are no standardised methods of applying diagnostic labels in general practice. GPs are faced with an array of alternative codes for the same or similar conditions and, in effect, practicing physicians have little faith in the accuracy of the ICD-9 diagnoses entered for prescription purposes; this can question the real value of diagnoses in the HSD.

A third critical issue is that most patients with chronic disease have more than one relevant diagnosis and the diagnosis recorded on the system for any particular visit may not be the primary reason for the visit. Furthermore, many physicians informally report paying little attention to the accuracy of the diagnosis recorded, relying instead on codes committed to memory from frequent use [38].

Nonetheless, we must highlight that comparative analyses have demonstrated the validity of the information gathered in the HSD [9]. Therefore, we believe that the prevalence of comorbidities in our asthmatic patients seems to be realistic because the use of a population-based, general practice database allows for study in a real-life clinical setting. Moreover, we must also admit, as stressed before, that this is a cross-sectional study and, consequently, the temporal relationship between clinically relevant comorbidities and asthma is unknown.

\section{Conclusion}

In conclusion, our study documented that numerous comorbidities are frequently associated with asthma. Therefore, the identification of comorbidities must become an integral part of the core management of asthma. As correctly stressed by BOULET [6], a systematic evaluation, not only of the presence of comorbid conditions, is necessary, but we have to ensure that these are also adequately treated/controlled so that their effect on asthma is minimised.

\section{STATEMENT OF INTEREST}

None declared.

\section{ACKNOWLEDGEMENTS}

This study is based on data from the Health Search Database owned by the Italian College of General Practitioners (Società Italiana Medici Generici, Florence, Italy). However, the interpretation and conclusions contained in this study are those of the authors alone. The original idea for the study was formulated by M. Cazzola, with subsequent advice from G. Bettoncelli and C. Cricelli, and statistical input from L. Calzetta who also performed the statistical analyses. M. Cazzola drafted the manuscript, with extensive contributions and revision from all authors. Access to the Health Search Database was offered free by the Italian College of General Practitioners.

\section{REFERENCES}

1 Sin DD, Anthonisen NR, Soriano JB, et al. Mortality in COPD: role of comorbidities. Eur Respir J 2006; 28: 1245-1257.

2 Wouters EF, Reynaert NL, Dentener MA, et al. Systemic and local inflammation in asthma and chronic obstructive pulmonary disease: is there a connection? Proc Am Thorac Soc 2009; 6: 638-647.

3 Fabbri LM, Luppi F, Beghé B, et al. Complex chronic comorbidities of COPD. Eur Respir J 2008; 31: 204-212.

4 Magnussen $\mathrm{H}$, Watz $\mathrm{H}$. Systemic inflammation in chronic obstructive pulmonary disease and asthma: relation with comorbidities. Proc Am Thorac Soc 2009; 6: 648-651.

5 Soriano JB, Visick GT, Muellerova H, et al. Patterns of comorbidities in newly diagnosed COPD and asthma in primary care. Chest 2005; 128: 2099-2107.

6 Boulet LP. Influence of comorbid conditions on asthma. Eur Respir J 2009; 33: 897-906.

7 World Health Organization. International Classification of Diseases. Manual of the International Statistical Classification of Diseases: Injuries and Causes of Deaths. 9th revision. Geneva, World Health Organization, 1977.

8 Lawrenson R, Williams T, Farmer R. Clinical information for research: the use of general practice databases. J Public Health Med 1999; 21: 299-304.

9 Filippi A, Bignamini AA, Sessa E, et al. Secondary prevention of stroke in Italy: a cross-sectional survey in family practice. Stroke 2003; 34: 1010-1014.

10 Zhang J, Yu KF. What's the relative risk? A method of correcting the odds ratio in cohort studies of common outcomes. JAMA 1998; 280: 1690-1691.

11 Schmidt OC, Kohlmann T. When to use the odds ratio or the relative risk? Int J Public Health 2008; 53: 165-167.

12 de Groot EP, Duiverman EJ, Brand PLP. Comorbidities of asthma during childhood: possibly important, yet poorly studied. Eur Respir J 2010; 36: 671-678.

13 Bousquet J, Vignola AM, Demoly P. Links between rhinitis and asthma. Allergy 2003; 58: 691-706.

14 Magnan A, Meunier JP, Saugnac C, et al. Frequency and impact of allergic rhinitis in asthma patients in everyday general medical practice: a French observational cross-sectional study. Allergy 2008; 63: 292-298.

15 Togias A, Zuberbier T, Baena-Cagnani CE, et al. Allergic Rhinitis and its Impact on Asthma (ARIA) 2008 update. Allergy 2008; 63: Suppl. 86, 8-160.

16 Price D. Asthma and allergic rhinitis: linked in treatment and outcomes. Ann Thorac Med 2010; 5: 63-64.

17 Rowe-Jones JM. The link between the nose and lung, perennial rhinitis and asthma - is it the same disease? Allergy 1997; 52: Suppl. $36,20-28$.

18 Togias A. Mechanisms of nose-lung interaction. Allergy 1999; 54: Suppl. 57, 94-105.

19 Dixon AE, Kaminsky DA, Holbrook JT, et al. Allergic rhinitis and sinusitis in asthma: differential effects on symptoms and pulmonary function. Chest 2006; 130: 429-435.

20 Havemann BD, Henderson CA, El-Serag HB. The association between gastro-oesophageal reflux disease and asthma: a systematic review. Gut 2007; 56: 1654-1664.

21 Harding SM, Richter JE. The role of gastroesophageal reflux in chronic cough and asthma. Chest 1997; 111: 1389-1402.

22 Cazzola M, Bettoncelli G, Sessa E, et al. Prevalence of comorbidities in patients with chronic obstructive pulmonary disease. Respiration 2010; 80: 112-119.

23 Schanen JG, Iribarren C, Shahar E, et al. Asthma and incident cardiovascular disease: the Atherosclerosis Risk in Communities Study. Thorax 2005; 60: 633-638.

24 Torén K, Lindholm NB. Do patients with severe asthma run an increased risk from ischaemic heart disease? Int J Epidemiol 1996; 25: $617-620$ 
25 Onufrak S, Abramson J, Vaccarino V. Adult-onset asthma is associated with increased carotid atherosclerosis among women in the Atherosclerosis Risk in Communities (ARIC) study. Atherosclerosis 2007; 195: 129-137.

26 Opolski M, Wilson I. Asthma and depression: a pragmatic review of the literature and recommendations for future research. Clin Pract Epidemiol Ment Health 2005; 1: 18.

27 Adams RJ, Wilson DH, Taylor AW, et al. Psychological factors and asthma quality of life: a population based study. Thorax 2004; 59: 930-935.

28 Roy-Byrne PP, Davidson KW, Kessler RC, et al. Anxiety disorders and comorbid medical illness. Gen Hosp Psychiatry 2008; 30: 208-225.

29 Appleton S, Ruffin R, Wilson D, et al. Asthma is associated with cardiovascular disease in a representative population sample. Obes Res Clin Pract 2008; 2: 91-99.

30 Rana JS, Mittleman MA, Sheikh J, et al. Chronic obstructive pulmonary disease, asthma, and risk of type 2 diabetes in women. Diabetes Care 2004; 27: 2478-2484.
31 Barnes PJ, Celli BR. Systemic manifestations and comorbidities of COPD. Eur Respir J 2009; 33: 1165-1185.

32 Shore SA. Obesity and asthma: possible mechanisms. J Allergy Clin Immunol 2008; 121: 1087-1093.

33 Mai XM, Böttcher MF, Leijon I. Leptin and asthma in overweight children at 12 years of age. Pediatr Allergy Immunol 2004; 15: 523-530.

34 Guler N, Kirerleri E, Ones U, et al. Leptin and asthma in overweight children at 12 years of age. J Allergy Clin Immunol 2004; 114: 254-259.

35 Al-Shawwa B, Al-Huniti N, Titus G, et al. Hypercholesterolemia is a potential risk factor for asthma. J Asthma 2006; 43: 231-233.

36 Lugogo NL, Kraft M, Dixon AE. Does obesity produce a distinct asthma phenotype? J Appl Physiol 2010; 108: 729-734.

37 Ebeling PR. Clinical practice. Osteoporosis in men. N Engl J Med 2008; 358: 1474-1482.

38 Katz A, Soodeen RA, Bogdanovic B, et al. Can the quality of care in family practice be measured using administrative data? Health Sero Res 2006; 41: 2238-2254. 